\title{
CFD simulation of dimpled sphere and its wind tunnel verification
}

\author{
Vojtěch Spálenský ${ }^{1, *}$ and Dalibor Rozehnal ${ }^{1}$ \\ ${ }^{1}$ University of Defence, Department of Air Force and Aircraft Technology, Kounicova 65, 66210 \\ Brno, Czech Republic
}

\begin{abstract}
Paper deals with problems of CFD simulating airflow over a dimpled spherical surface and its verification by the wind tunnel measurement. The low-cost simulation approach was applied to be run on a common PC using the commercial software ANSYS CFX. The wind tunnel testing has been performed in the laboratory of aerodynamics at the Department of Air Force and Aircraft Technology of the University of Defence. Measured results of the drag coefficient versus the Reynolds number for smooth and dimpled spheres were compared and discussed. Presented simulation corresponds adequately to the experimental results. It can be stated that the CFD simulation is suitable for simulating the flow over the dimpled surfaces similar to sphere.
\end{abstract}

\section{Introduction}

The surface structure influences the air flow over objects of various shapes. Due to reducing the aerodynamic drag, the dimpled surface structure may be used. The typical example of using the dimpled surface is a golf ball. Dimpled golf balls have been used for almost a century. Empirical observations had shown that the dimpled balls travel farther due to reduction in pressure drag.

Dimpled surfaces reducing the aerodynamic drag are often applied in the design of sport equipment. There is a large potential in using of dimpled surfaces for the aerodynamic drag reduction of various objects, where the laminar-turbulent transition is inducted $[1,2]$. Nowadays, the research of dimple's aerodynamics is also focused on the study of increasing the heat transfer rate [3,4].

Most of the current CFD simulations of dimpled surfaces use the high demand turbulence models like DNS (direct numerical simulation), or LES (large eddy simulation) $[3,4]$. These numerical models require a very fine mesh causing high computational demands of these applications.

The paper is focused on the study of the possibility of the low-cost, three-dimensional CFD simulation of flow around a dimpled surface. A common PC and commercial software ANSYS CFX were used. Simulation model was tested on dimpled sphere and was verified by a wind tunnel measurement.

\footnotetext{
*Corresponding author: vojtech.spalensky@unob.cz
} 


\section{Wind tunnel testing}

For the validation of the simulation model, it is necessary to obtain experimental data for both smooth and dimpled spherical surfaces. The wind tunnel testing has been performed in the laboratory of aerodynamics at the Department of Air Force and Aircraft Technology of the University of Defence. The wind tunnel is the closed return one with a diameter of the testing section $600 \mathrm{~mm}$ and the range of free stream velocities from 2 to $45 \mathrm{~m} / \mathrm{s}$.

The wind tunnel model of the dimpled sphere has diameter $D=216 \mathrm{~mm}$ the surface of which is provided with 392 circular dimples of the diameter $d=14.8 \mathrm{~mm}$ and depth $h=1.5$ $\mathrm{mm}$, so the $h / d$ ratio is about 0.1 (see Fig. 1).

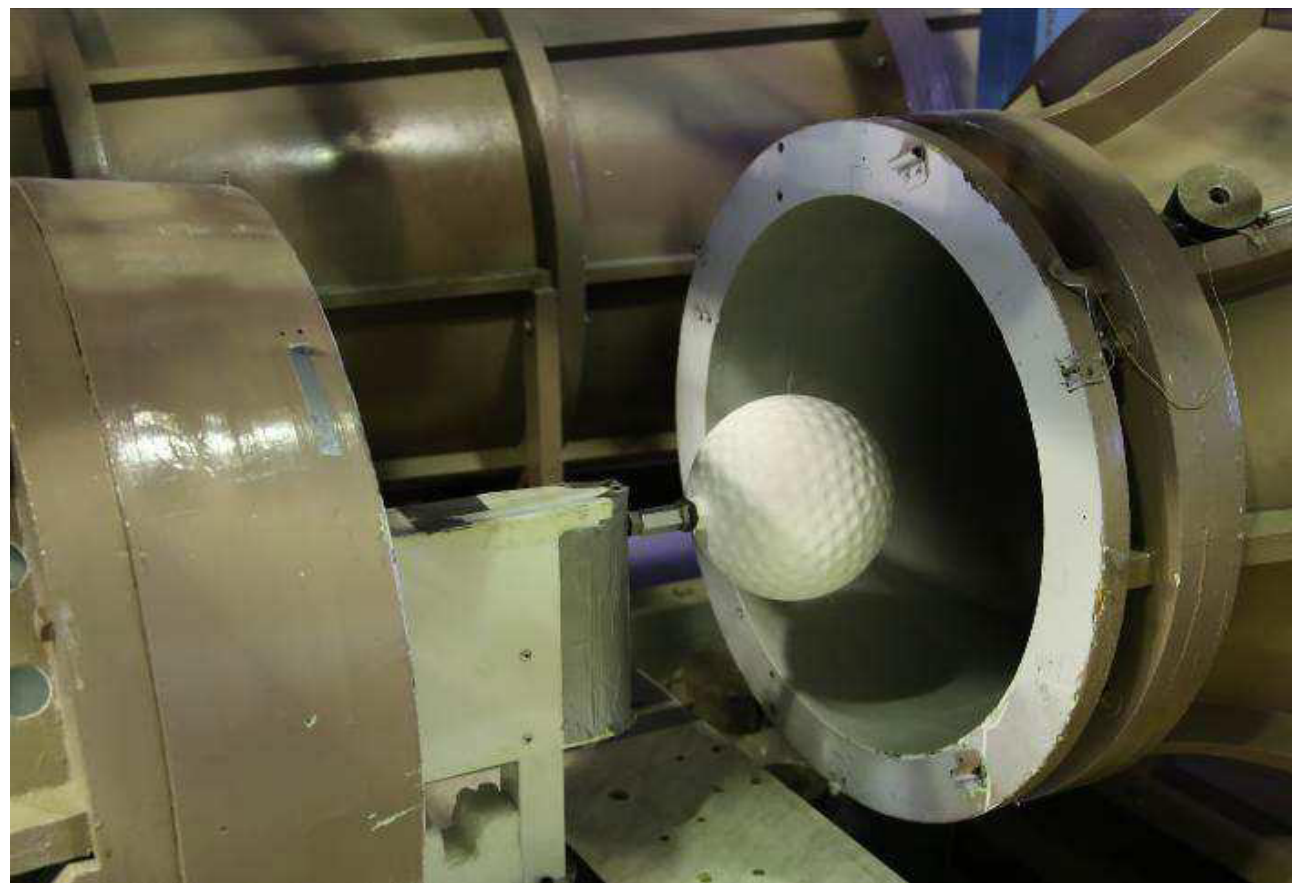

Fig. 1. Model of the dimpled sphere in the wind tunnel section.

The aerodynamic force measurement system is based on two strain gauges. The analogue signals from them are processed by a computer. Measuring network is mostly build on National Instrument hardware and LAB-VIEW software.

The drag force was measured for various free stream velocity in the range of Reynolds numbers from 50000 to 420000 . The lower limit of the measurement range is given by the capabilities of a force measuring instrument. The upper measurement range limit is given by the maximum velocity of the free stream in used wind tunnel. 


\section{Results of testing and analysis}

Results of testing in the form of drag coefficient $C_{D}$ versus the Reynolds number Re for dimpled and smooth spheres are given in Fig 2. Here, it is seen the substantial difference between drag curves for both observed objects.

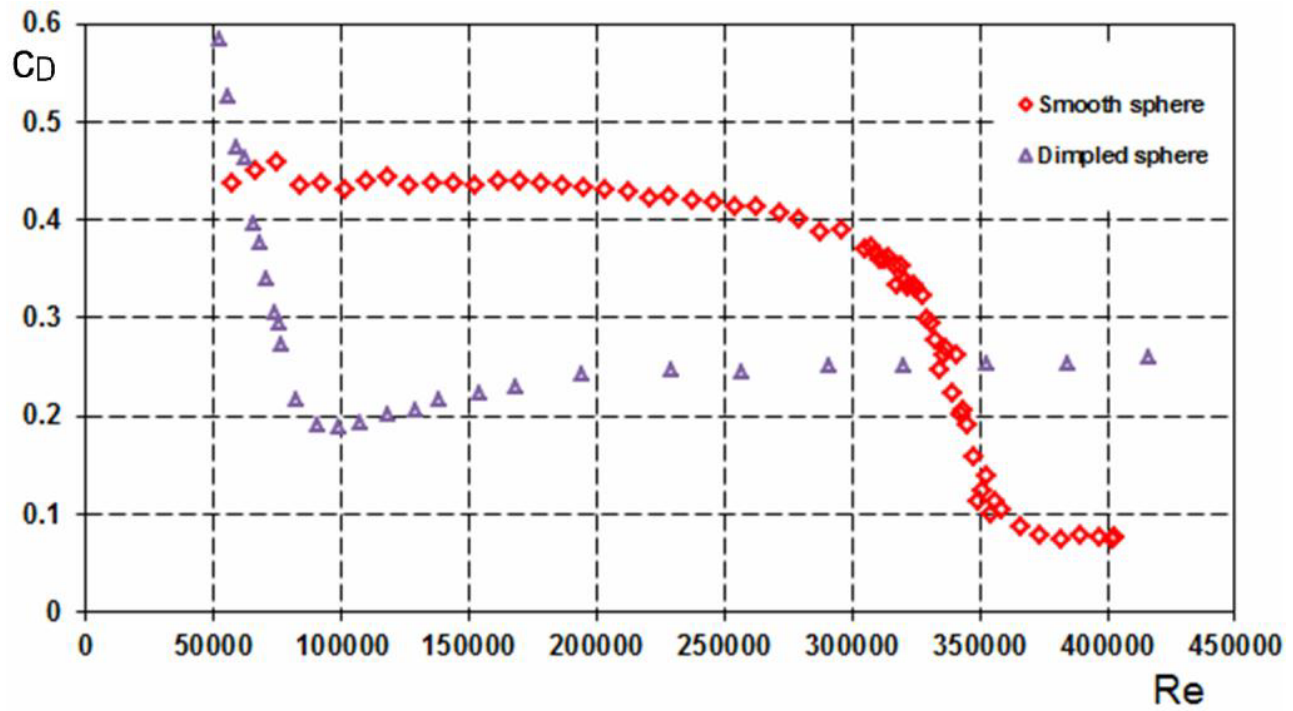

Fig. 2. Measured dependence of drag coefficient on Reynolds number for smooth and dimpled spheres.

In the case of smooth sphere the drag coefficient is nearly constant with the value about $C_{D}=0.42$ in range of Re from 50000 to 300000 , then there is a sudden decrease of $C_{D}$ for Re between 300000 and 350000 . The value of the drag coefficient drops to one quarter, i.e. under $C_{D}=0.1$.

The same dependence of the drag coefficient on the Reynolds number for dimpled sphere is quite different. This decline in drag coefficient is between Re 50000 and 100000 . The minimum value of the drag coefficient is $C_{D}=0.195$. Then between $\operatorname{Re} 100000$ and 200000 the drag coefficient slightly increases to $C_{D}=0.27$. Almost constant value up to the maximum velocity corresponding to the Reynolds number 420000 is observed next.

Finally, it can be stated that the drag coefficient for the dimpled sphere is lower than for the smooth one in the range of Reynolds numbers between 60000 and 340000 . However, the smooth sphere has lower drag coefficient than the dimpled one for Reynolds numbers above 340000 .

Presented experimental results correspond very well with the results measured by [5] for smooth and dimpled golf balls.

\section{Results of testing and analysis}

The three-dimensional CFD simulation, based on Reynolds averaged Navier-Stokes equations by the CFD software ANSYS CFX 14.5 [6], was applied to simulate the flow over the dimpled and smooth spherical surfaces. The simulation was performed as a lowcost, on the standard notebook HP Pavilion $\mathrm{g} 7$ with the Intel core i7 processor and 8 GB 
operational memory. The computing time of the simulation for one flow of velocity took about twenty hours.

The tetra-prism mesh was used, because this one has proved to be most suitable for the simulation of flow over a single dimple [7] and also this kind of mesh is better for the simulation of a wake zone.

In the case of flow over the single dimple, with the increase in velocity of the free stream the flow character is changing from two symmetrical vortices to one asymmetric vortex. This change occurs between free stream velocities about $10 \mathrm{~m} / \mathrm{s}$ and $20 \mathrm{~m} / \mathrm{s}$ (see Fig. 3). That matched the observations [8] and also simulations [1,2]. This change of the flow character is obvious, when streamlines in simulations that use tetra-prismatic mesh, as shown in Fig. 3, are compared. There are no further radical changes in a flow with the next velocity increase. An asymmetric vortex has two possible stabile orientations. Orientation of the vortex depends on the mesh parameters.

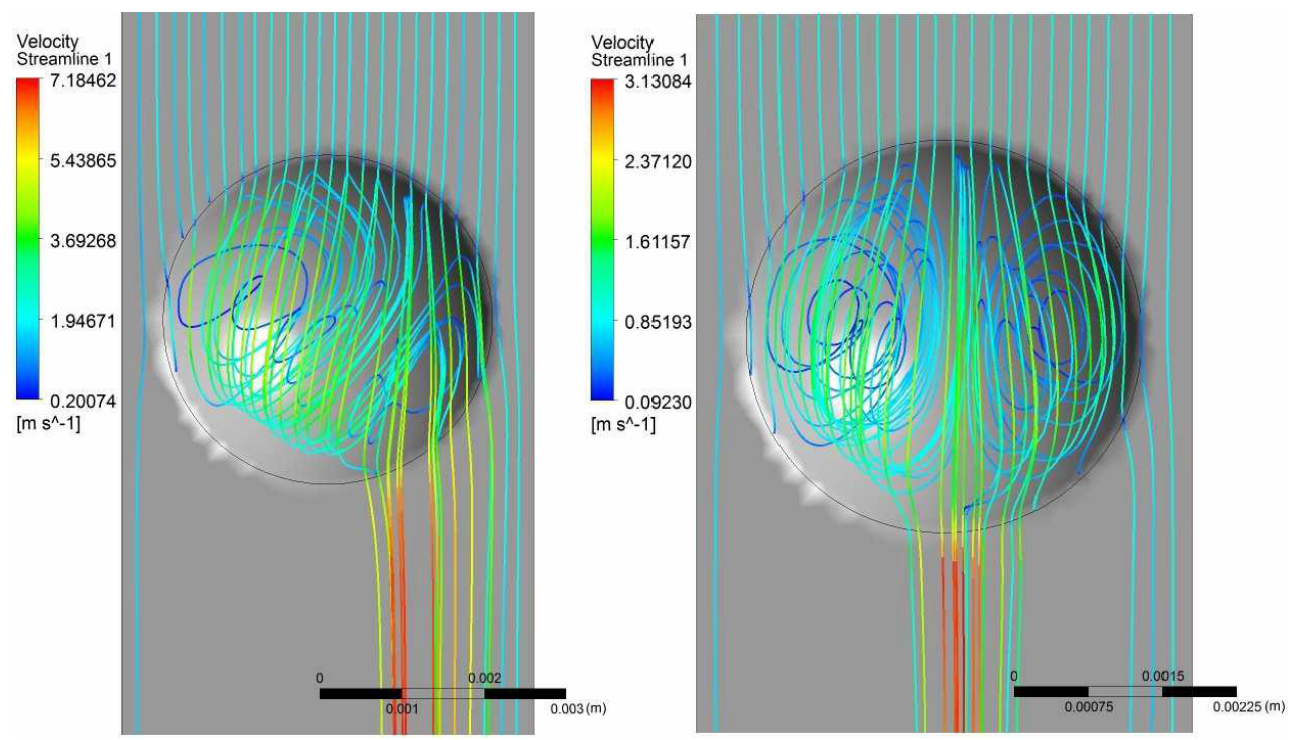

Fig. 3. Comparison of streamlines simulation of flow over single dimple for two free stream velocities: left $20 \mathrm{~m} / \mathrm{s}$ and right $10 \mathrm{~m} / \mathrm{s}$. Tetra-prism mesh was used for simulation.

The geometry for the simulation of flow over the dimpled sphere is the same as the geometry of the wind tunnel model. Due to reducing the computational demands, the only a quarter of the dimpled sphere was simulated. The domain is chosen as the quarter of a cylinder of length $4 \mathrm{~m}$ and radius $1 \mathrm{~m}$.

The position of the sphere center is in the distance of $1.5 \mathrm{~m}$ from the inlet section. The computational mesh was generated by using the mesh generator ICEM CFD [9]. The mesh consists of 8000000 cells and the overall view of the mesh is shown in Fig. 4.

A near surface region is composed by 32 prismatic layers. The surface mesh around the sphere is formed by 150000 cells. The density of cells on the surface is constant. Detail of the computational mesh near the spherical surface is shown in Fig. 5. 


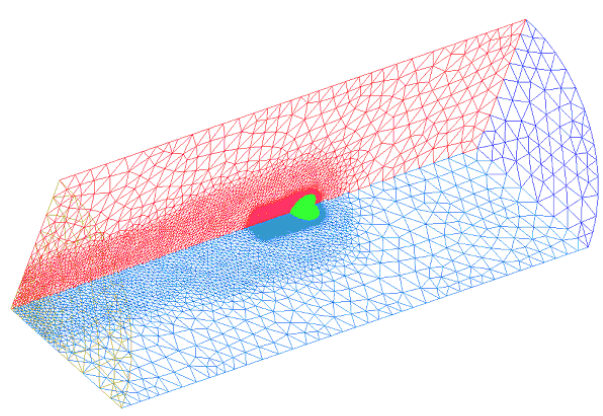

Fig. 4. Overall view of computational mesh.

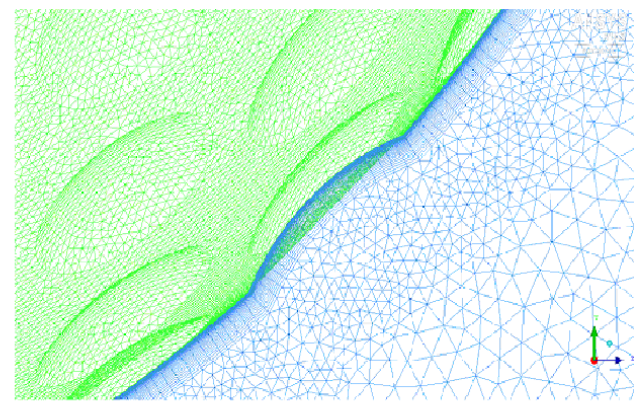

Fig. 5. Detail of computational mesh near surface.

The physical model and the boundary conditions of the CFD simulation were the following:

-The steady state simulation, due to the lower computational demands. Although the flow over a single dimple is of unsteady state [7, 8], I try to examine whether such condition is acceptable.

-The uncompressible fluid (air) of constant density, with no heat transfer.

- The ambient air temperature is $25^{\circ} \mathrm{C}$.

- The reference pressure is $101325 \mathrm{~Pa}$ and the relative pressure on the outlet is $0 \mathrm{~Pa}$.

-Inlet free stream turbulence intensity is considered $5 \%$

- The used turbulence model is the shear stress transport model and the laminarturbulent boundary layer transition onset follows the gamma-theta model [10].

-The simulation was performed for seven free stream velocities: 5, 6.5, 7, 8, 9, 10 , and $15 \mathrm{~m} / \mathrm{s}$.

-The symmetry was set on sides of the domain, as I simulated only the quarter of observed object.

-The surface of the sphere is considered as the smooth wall.

-The domain outer cylindrical wall is set up as the free slip wall.

-The convergence criterion was associated with a stabilization of the drag force.

\section{Results of simulation and analysis}

There are presented some examples of CFX POST outputs for smooth and dimpled spheres. The velocity distributions around the observed streamlined objects are shown in Fig. 7.

For the smooth sphere at high Reynolds number $\mathrm{Re}=647000$, the turbulent separation takes place farther toward the rear of the sphere, at a position about $118.5^{\circ}$ from the front stagnation point, as seen in Fig. 7a). This causes the sudden drop in the drag coefficient $C_{D}$, to a minimum of about 0.1 (see Fig. 2).

In the range of Reynolds numbers from 50000 to 300 000, the drag coefficient of smooth sphere remains almost constant at about 0.4 (see Fig. 2). The flow separates at a position about $83^{\circ}$ from the front stagnation point, and there is a fully developed turbulent wake, as seen in Fig. 7b). For the simulation of the flow around the smooth sphere, the same CFD computational model was applied.

In the case of the dimpled sphere, the flow pattern around this object is nearly the same (Fig. 7c) in the whole examined range of Reynolds numbers from 65000 to 209000 [7]. However, there is a significant difference between the flow patterns around the smooth and 
dimpled spheres. For the dimpled sphere at $\mathrm{Re}=209$ 000, the flow separates at a position about $90^{\circ}$ from the front stagnation point, as seen in Fig. 7c), and the drag coefficient is about 0.24 .

Results of simulation for flow around the dimpled sphere in terms of integral characteristics as the dependence of drag coefficient versus Reynolds number are presented in Fig. 6. The comparison of calculated and measured values shows that the maximum difference is about $30 \%$, but for Reynolds numbers over 130000 , the difference is lower than $10 \%$.

These differences are probably caused by an inadequacy in the domain geometry when some dimples are divided. But the more significant is an inadequacy in the physical model, which does not allow the detailed simulation of transition processes in a zone of the sudden drop in the drag coefficient.

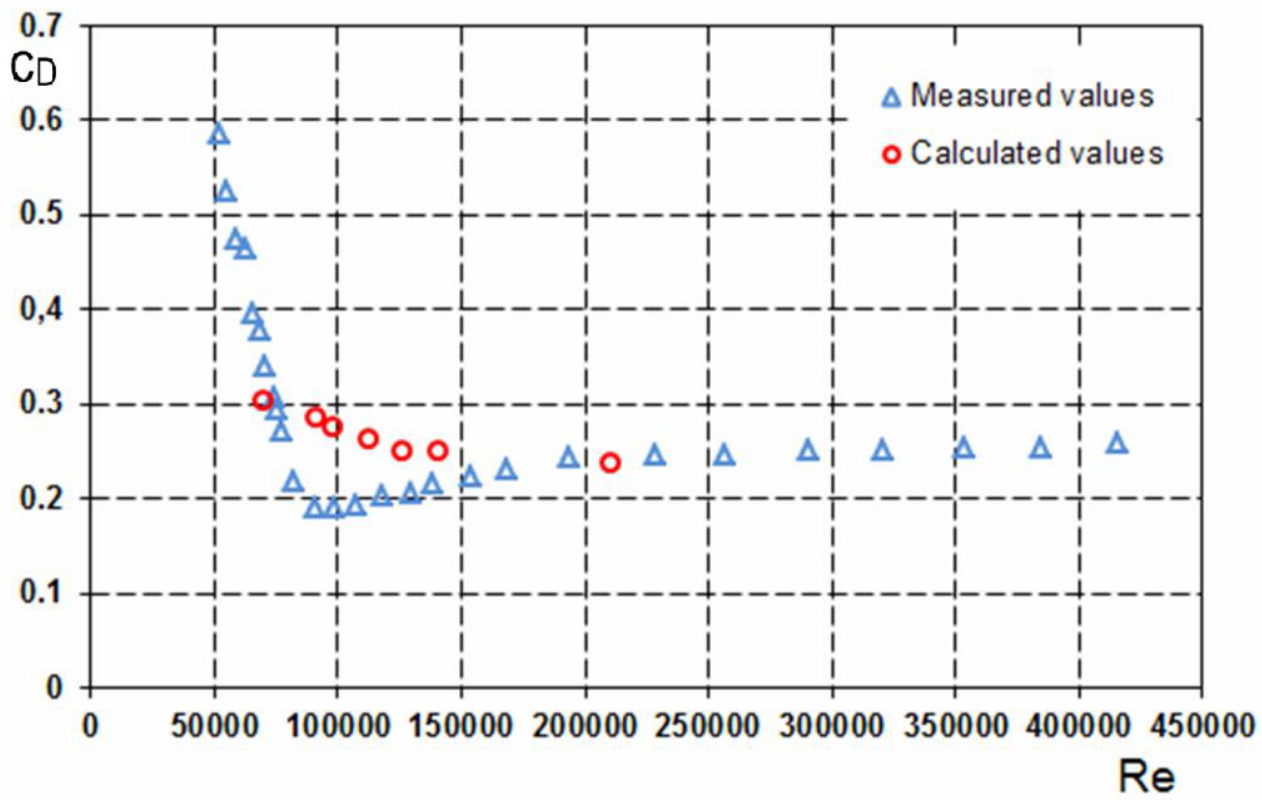

Fig. 6. Comparison of calculated and measured drag coefficients for dimpled sphere. 

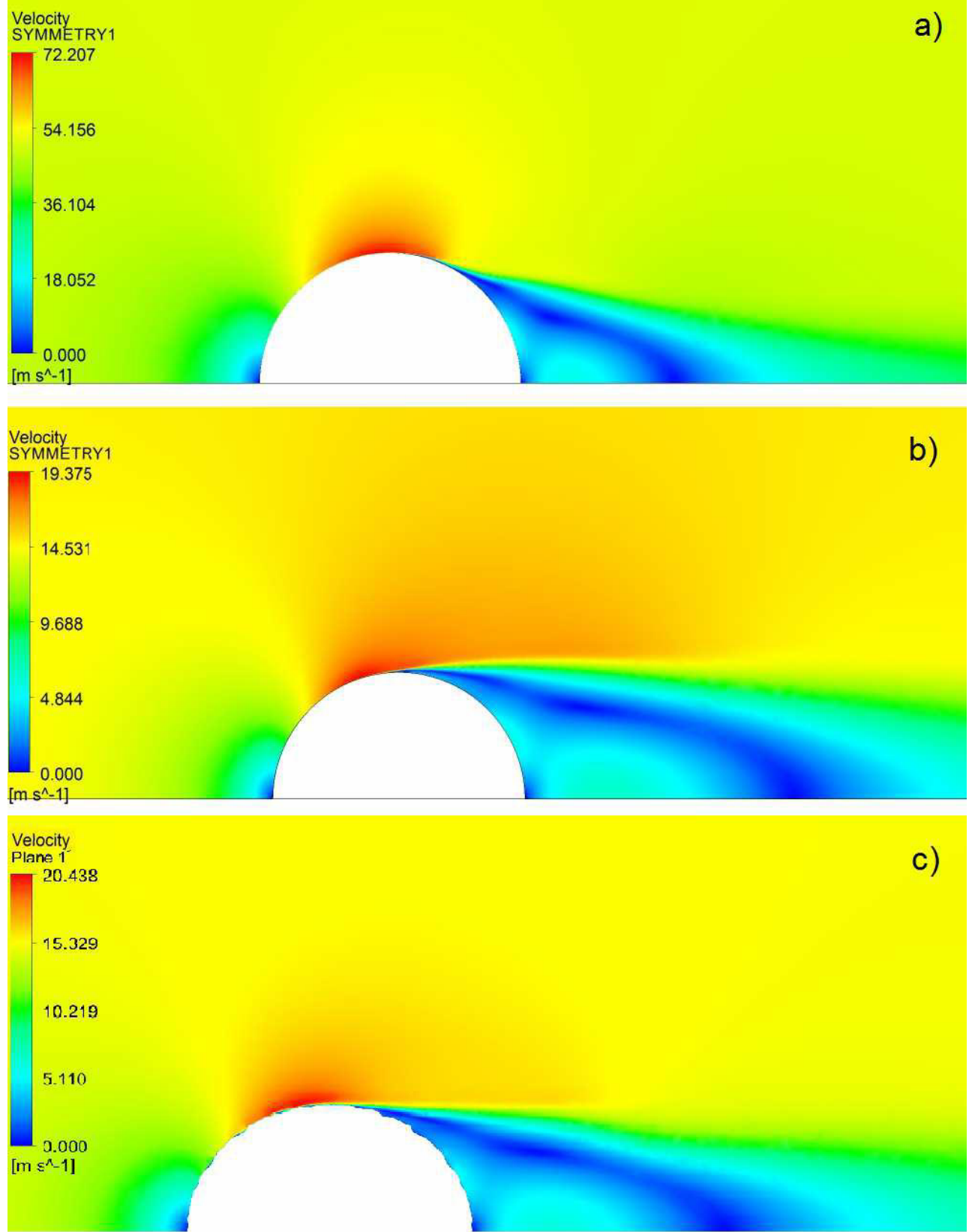

Fig. 7. Comparison of velocity distributions around observed spheres: a) smooth $\operatorname{Re}=647000, b$ ) smooth $\mathrm{Re}=194000, \mathrm{c}$ ) dimpled $\mathrm{Re}=209000$.

\section{Conclusions}

This easy-to-use CFD model has been developed for the simulation of a flow over the dimpled rounded surfaces. The simulation model is suitable for fast and low-cost calculations by using a standard PC. 
Presented model is mainly suitable as a criterion for deciding whether to use smooth or dimpled surfaces and evaluate conditions and application areas of structured surfaces for a drag reduction.

Usability of this model has been already confirmed on the dimpled cylinder simulation, where it was used even for optimization [11].

\section{References}

[1] S. A. Isaev, N. V. Kornev, A. I. Leontiev, and E. Hassel, Influence of the Reynolds number and the spherical dimple depth on turbulent heat transfer and hydraulic loss in a narrow channel. int.j.heat mass tran 53, 1-3, 178-197 (2010)

[2] J. Turnow, N. V. Kornev, S. A. Isaev, and E. Hassel, Vortex mechanism of heat transfer enhancement in a channel with spherical and oval dimples. int.j.heat mass tran 47, 3, 301$313(2011)$

[3] H. Schlichting, Boundary-Layer Theory (New York: Mc Graw-Hill, 1979)

[4] C. E. Smith, Direct Numerical Simulation of the Flow over a Golf Ball [Dissertation], (Phoenix: Arizona State University, 2011)

[5] P. W. Bearman and J. K. Harvey, Golf Ball Aerodynamics, AeQ, 112-122 (1976)

[6] ANSYS CFX, Reference Guide, Release 12.0, ANSYS, Inc. (2009)

[7] V. Spálenský, CFD Simulation of the flow over the dimpled surface [Thesis, in Czech], (Brno, University of Defense, 2014)

[8] V. I. Terekhov, S. V. Kalinina, Y. M. Mshvidobadse, Heat transfer coefficient and aerodynamic resistance on a surface with a single dimple. $\mathrm{j}$ enhanc heat tranf. 4, 131-145 (1997)

[9] ANSYS ICEM CFD 11.0, Tutorial Manual, ANSYS, Inc., January (2007)

[10] R. B. Langtry and F. R. Menter, Transition Modeling for General CFD

Applications in Aeronautics, AIAA Paper (2005-522), 43rd AIAA Aerospace Science Meeting and Exhibit, Reno, Nevada: AIAA, (2005)

[11] V. Spálenský, Optimization of dimples geometry on surface of cylinder [Thesis, in Czech], (Brno, University of Defense, 2016) 